\title{
Enhanced Adaptively Space-Time-View Error Concealment for 3D Multi-View Video Transmission over Noisy Channels
}

\author{
Walid El-Shafai \\ Department of Electronics and Communications Engineering, Faculty of Electronic Engineering, Menoufia \\ University, Menouf, 32952, Egypt.
}

\begin{abstract}
Decoding of 3D multi-view video coded bit-streams that are transmitted over noisy channels can suffer from error propagation in the space, time and view domains. To improve the quality of reconstructed $3 D$ video, we propose adaptive Error Concealment (EC) algorithms to conceal the erroneous blocks of intra-coded and inter-coded frames. Our proposed algorithms adapt to the motion characteristics of the $3 D$ video and to the error patterns. We propose three adaptive EC algorithms for intra-frames, which are Adaptive Time Domain Error Concealment (ATDEC), Adaptive Space Domain Error Concealment (ASDEC) and a hybrid Adaptive Space-Time Domain Error Concealment (ASTDEC). We also propose three adaptive EC algorithms for interframes, which are Adaptive Inter-View Domain Error Concealment (AIVDEC), Adaptive Time Domain Error Concealment (ATDEC) and joint Adaptive Time and Inter-View Domain Error Concealment (ATIVDEC). Our simulation results show that the proposed adaptive EC algorithms can significantly improve the objective and subjective $3 D$ video quality transmitted over noisy channels.
\end{abstract}

Keywords - Multi-view Coding, 3D Video, Error Concealment, Intra Frame, Inter Frame, Spatio-Temporal and Inter-view Correlation.

\section{INTRODUCTION}

Multi-view video has received a huge attention lately and is expected to quickly replace traditional 2D video in different applications. In Multi-view Video Coding (MVC), the original video content is a group of video sequences captured for the same scene by multiple cameras at the same time and from different viewpoint angles. Multi-view video sequences exhibit high inter-view correlation, in addition to the spatio-temporal correlations within each view. Error Concealment (EC) exploits this advantage of inter-view and intra-view correlations to conceal erroneous packets or frames [1]. EC algorithms are attractive as they neither require encoder changes nor they result in increasing the transmission delay or bit rate [2, 3]. In our previous work [4], we proposed different EC algorithms to recover the lost Macro-Blocks (MBs) of intra and inter coded frames by exploiting the temporal, spatial and inter-view correlations between frames and views.

In [4], temporal EC deploys External Block Boundary Matching Algorithm (EBBMA) [5], and spatial and inter-view EC deploy Weight Pixel Averaging Algorithm (WPAA) [6]. To further improve the quality of decoded 3D video, we propose, in this paper, adaptive EC algorithms that adapt to the motion characteristics of the received 3D MVC video sequence (slow or fast moving video) and to the locations of error patterns. In this work, we propose adaptive EC algorithms for 3D MVC sequences generated by using the Joint MVC (JMVC) reference software based on H.264/AVC [3]. In this paper, temporal EC adapts and selects between three different EC modes which are Frame Temporal Replacement Algorithm (FTRA), EBBMA, and Decoder Motion Vector Estimation Algorithm (DMVEA) [5]. Also, spatial and inter-view EC adapt and choose between two different EC modes which are WPAA and Directional Interpolation EC Algorithm (DIECA) [6].

The rest of this paper is organized as follows: Section 2 presents the proposed error concealment algorithms for intra- frames and inter-frames, Section 3 presents our experimental simulation results and Section 4 concludes the paper.

\section{Proposed AdAPTIVE Mve Ec Algorithms}

Fig. 1. shows a high-level flow chart of our proposed adaptive space-time-view MVC EC decoder algorithm at the decoder. Fig. 1 shows when each of the component algorithms should be invoked. In this section, we explain in details each of the component algorithms of our proposed MVC EC algorithm. 


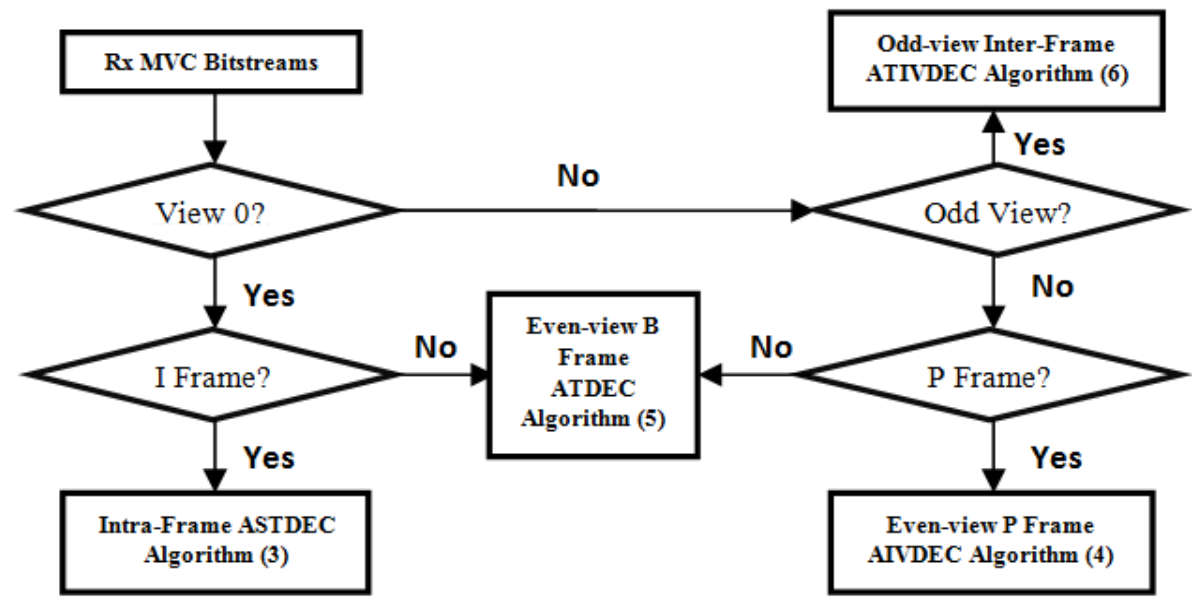

Fig.1 Adaptive Space-Time-View MVC EC Algorithms.

\subsection{Intra-Frame Space-Time Error Concealment}

Due to the predictive coding structure of MVC (cf. Fig. 2 [7]), EC of MVC intra-frames is not only important for improving the video quality of decoded intra-frames but is also important for efficient reconstruction of inter-frames in the subsequent frames and views.

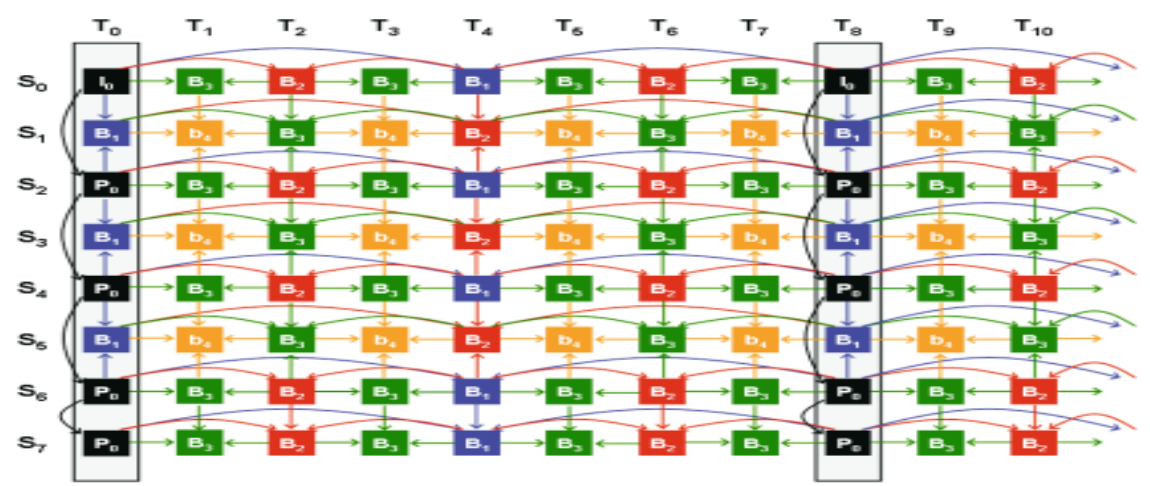

Fig. 2 Efficient Prediction Structure for MVC Proposed [7].

The simplest temporal EC technique is FTRA method [5], which is suitable and acceptable for static and very slow 3D MVC sequences, where the lost MBs are replaced by the MBs located at the same spatial location in the reference frame. On the other hand, the more sophisticated temporal EC techniques are the EBBMA and the DMVEA methods [5].

In EBBMA algorithm, the decoder determines the Motion Vectors (MVs) for error-free neighboring pixels in the reference frames which are adjacent to the lost MBs and replace the lost MBs with the MBs in the reference frames that minimize the boundary error to the correctly received adjacent MBs. So, it only uses the outer borders of the reference MBs to check the highly correlated neighborhood MVs and the neighborhood MBs can be predicted using Disparity Vectors (DVs) values. In DMVEA algorithm, the decoder estimates the MVs of the lost blocks by using a full search in the reference frames, and it is useful in identifying the replacing MBs that minimize the boundary distortion error.

In EBBMA and DMVEA algorithms, the best matching MBs are searched in the reference frames by reducing a Sum of Absolute Differences (SAD) [8], between boundary pixels of the corrupted MBs in the current frame and their corresponding correlated pixels in the reference frame. Generally, DMVEA presents better EC performance than EBBMA. However, depending on the patterns or locations of the corrupted MBs, EBBMA may achieve approximately the same visual quality as compared with DMVEA. Therefore, the SAD value can be a suitable method for comparing the performance of EBBMA and DMVEA. So, on general the DMVEA algorithm leads to improvement in concealment performance and is considered as more powerful and effective than the EBBMA algorithm.

Consider an intra-frame with a missing Macro-Block (MB) as shown in Fig. 3. The vectors $\mathbf{P}_{\mathbf{U 1}}, \mathbf{P}_{\mathbf{U} 2}$, $\mathbf{P}_{\mathbf{R} 1}, \mathbf{P}_{\mathrm{R} 2}, \mathbf{P}_{\mathrm{B} 1}, \mathbf{P}_{\mathrm{B} 2}, \mathbf{P}_{\mathrm{L} 1}$, and $\mathbf{P}_{\mathrm{L} 2}$ consist of the outside boundary pixels of the upper, right, bottom and left sides of the missing $\mathrm{MB}$, respectively. The upper, right, bottom and left inner boundary pixels of the candidate $\mathrm{MB}$ 
are represented by the vectors $\mathbf{X}_{\mathrm{U} 1}, \mathbf{X}_{\mathrm{U} 2}, \mathbf{X}_{\mathbf{R} 1}, \mathbf{X}_{\mathbf{R} 2}, \mathbf{X}_{\mathbf{B} 1}, \mathbf{X}_{\mathbf{B} 2}, \mathbf{X}_{\mathrm{L} 1}$ and $\mathbf{X}_{\mathbf{L} 2}$, respectively [5]. The MVs between the adjacent sub-blocks and their matching blocks can be calculated by the following equations (1-6).

$$
\begin{array}{rrr}
\widehat{\mathrm{X}}=\arg _{X_{\mathrm{U} 1} x_{\mathrm{U} 2}, \cdots \cdots X_{\mathrm{L} 2}} \min \varepsilon^{2} & \text { (1), } & \varepsilon^{2}=\varepsilon_{\mathrm{U}}^{2}+\varepsilon_{\mathrm{R}}^{2}+\varepsilon_{\mathrm{B}}^{2}+\varepsilon_{\mathrm{L}}^{2} \\
\varepsilon_{U}^{2}=\sum_{i=1,2}\left\|\left(X_{U_{i}}-P_{U_{i}}\right)\right\|^{2} & (\text { (3), } & \varepsilon_{R}^{2}=\sum_{i=1,2}\left\|\left(X_{R_{i}}-P_{R_{i}}\right)\right\|^{2} \\
\varepsilon_{B}^{2}=\sum_{i=1,2}\left\|\left(X_{B_{i}}-P_{B_{i}}\right)\right\|^{2} & \text { (5), } & \varepsilon_{L}^{2}=\sum_{i=1,2}\left\|\left(X_{L_{i}}-P_{L_{i}}\right)\right\|^{2}
\end{array}
$$
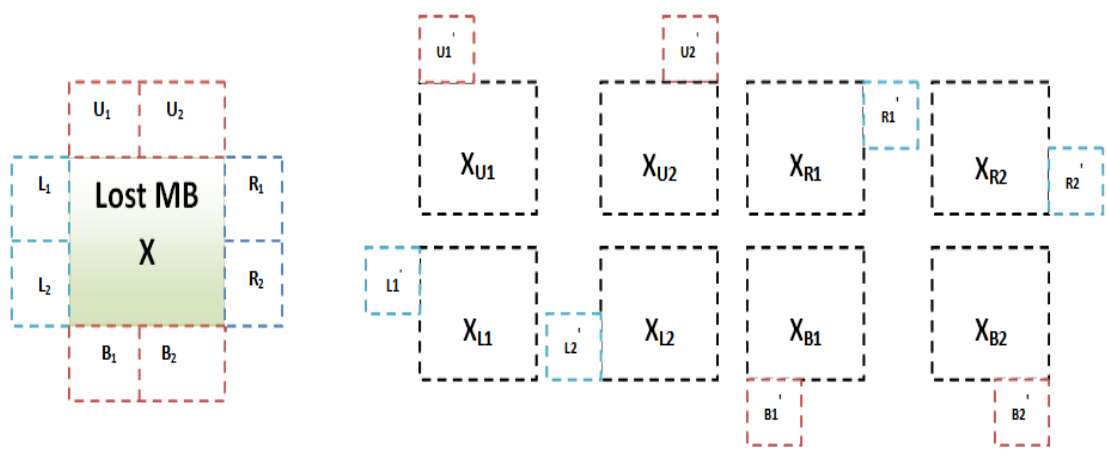

Fig. 38 Sub-Blocks Adjacent to the Lost MB and their Matching Sub-Blocks and Candidate Blocks in the Previous Frame.

The hybrid space-time EC algorithm invokes the adaptive time domain and space domain EC algorithms as detailed below, and illustrated in Fig. 4.

Algorithm 1: Intra-Frame Adaptive Time Domain EC (ATDEC) Algorithm

1. Find the locations of the lost MBs inside $\mathbf{I}$ frame.

2. Apply Scene Change Detection Algorithm [6], to decide "Is the received 3D MVC video fast or slow moving video?".

3. Select between the three ATDEC algorithms (FTRA or EBBMA or DMVEA) depending on step (2), as noticed in Fig. 4:

If the received video is a slow moving video, Then

- Apply the FTRA algorithm.

- Replace the lost MBs by the MBs located at the same spatial positions in the previous frame.

If the received video is a fast moving video, Then

- Find the $8 \times 8$ adjacent sub-blocks to the lost MB i.e., $\mathbf{U}_{\mathbf{1}}, \mathbf{U}_{\mathbf{2}}, \mathbf{R}_{\mathbf{1}}, \mathbf{R}_{\mathbf{2}}, \mathbf{B}_{\mathbf{1}}, \mathbf{B}_{\mathbf{2}}, \mathbf{L}_{\mathbf{1}}$ and $\mathbf{L}_{\mathbf{2}}$ and their matching blocks U1', U2', R1', R2', B1', B2', L1' and L2' in the reference frame as shown in Fig. 3.

- Calculate the MVs between the adjacent sub-blocks and their matching blocks by using (3-6) for the two proposed EBBMA and DMVEA algorithms.

- Select the candidates MBs that give the smallest SAD value given by (2).

- Select between the EBBMA algorithm and the DMVEA algorithm depending on SAD value; If the calculated SAD value is less than or equal to the predetermined threshold, e.g. 0.2, apply EBBMA algorithm, otherwise apply DMVEA algorithm.

- Average MVs values of the candidates MBs.

- Replace the lost MBs with the candidates MBs by using the averaged calculated value.

The WPAA and DIECA algorithms are used for spatial and inter-view EC. WPAA algorithm conceals the damaged pixels using the horizontal and vertical pixels in the neighboring blocks. DIECA algorithm conceals the damaged pixels by calculating the object edge direction from the neighboring blocks, where the object edge direction with the largest magnitude is chosen as the direction to be used to conceal the damaged MBs [9].

DIECA algorithm introduces two main problems which are: (1) it may fail when concealing the damaged MBs that are at the corner of the frame, and (2) it may fail when concealing the lost MBs that are at the edge of the frame. So, our proposed ASDEC algorithm is able to choose the most appropriate EC algorithm to use, either DIECA or WPAA algorithm. In the following algorithm, we propose to apply WPAA algorithm and drop DIECA algorithm if the damaged MBs are at the corner or at the edge of the frame. 


\section{Algorithm 2: Intra-Frame Adaptive Space Domain EC (ASDEC) Algorithm}

1. Find the locations of the lost MBs inside I frame.

2. Select between the two ASDEC algorithms (WPAA or DIECA), as indicated in Fig. 4, to find the matching pixels surrounding the lost MB's pixels depending on the location of the lost MBs.

a. If "the lost MBs are at the edge or at the corner of the frame"

Else

- Apply the WPAA algorithm.

- Apply the DIECA algorithm.

b. Calculate the DVs between pixels inside the lost MBs and pixels surrounding the lost MBs for the WPAA algorithm or DIECA algorithm.

3. Find the average value of the candidates DVs of the calculated pixels found in previous step.

4. Replace the lost MBs with that calculated in the last step.

Algorithm 3: Intra-Frame Adaptive Space-Time Domain EC (ASTDEC) Algorithm

1. Find the locations of the lost MBs inside I frame.

2. Find the temporal candidates MBs using ATDEC Algorithm.

3. Find the spatial candidates MBs using ASDEC Algorithm.

4. Average DVs and MVs values of the candidates MBs which are founded in the previous steps (2) and (3).

5. Apply Scene Change Detection Algorithm, to check if Temporal information $>$ Spatial information or vice versa.

6. Set appropriate coefficient values to averaged values of MVs and DVs ( avg (MVs) and avg (DVs), respectively) depending on Scene Change Detection Algorithm [6]:

If Temporal information $<$ Spatial information

Else

- $\quad$ Candidate $\mathrm{MB}=1 / 3$ avg $(\mathrm{MVs})+2 / 3$ avg (DVs).

- $\quad$ Candidate $\mathrm{MB}=2 / 3$ avg (MVs) $+1 / 3$ avg (DVs).

7. Replace the lost MBs with the candidates MBs calculated using the weighted average of MVs and DVs as in last step.
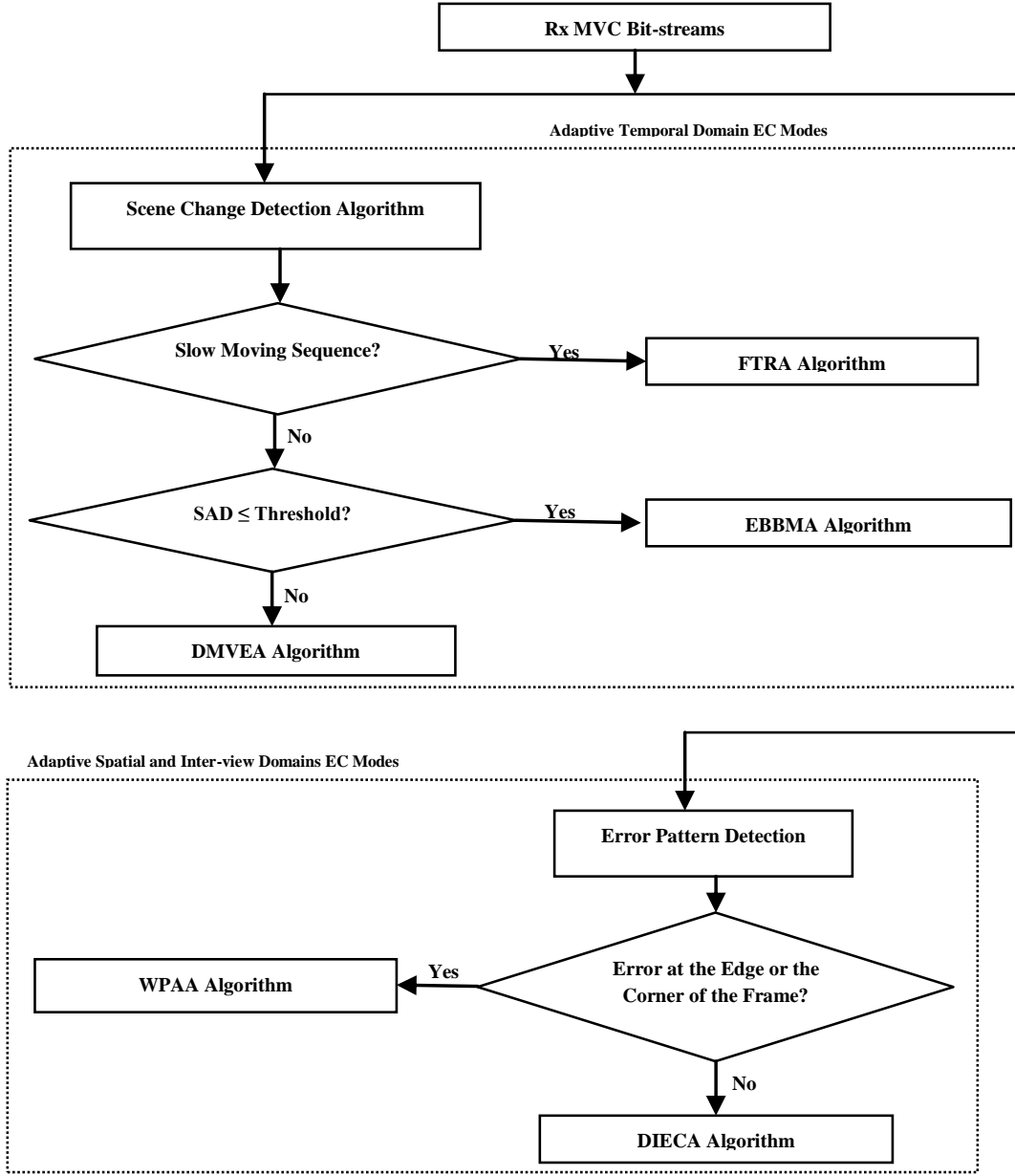

Fig. 4 Flow Chart of Selection Modes Space-Time-View EC Algorithms. 


\subsection{Inter-Frame Time Inter-View Error Concealment}

EC of inter-view frames (B and P frames) should be different across views based on the MVC prediction structure of Fig. 2. In even views (e.g. $S_{2}, S_{4}$ and $S_{6}$ ), the first frame is concealed using left inter-view frames as reference frames whereas other frames are concealed using the previous and following frames in the same view as the reference frames. In odd views (e.g. $S_{1}, S_{3}$ and $S_{5}$ ), EC is done using the previous and following frames in the same view as well as the left and right frames in the adjacent views as the reference frames. Thus, except the first frames, there are two candidate blocks for EC of lost MBs of inter-frames in even views, and four candidates for EC of lost MBs of inter-frames in odd views. This is also illustrated in Fig. 2. Our proposed adaptive inter-frame EC algorithm has three EC modes as described below:

\section{Algorithm 4: Even-view P Frame Adaptive Inter-View Domain EC (AIVDEC) Algorithm}

1. Find the locations of the lost MBs inside $P$ frame.

2. Apply WPAA Algorithm or DIECA Algorithm, as illustrated in Fig. 4, to find the matching pixels inside the left frame depending on the locations and the pattern of the lost MBs.

3. Apply step 2 in the Algorithm 2 in the left frame.

4. Average DVs values of the candidates MBs calculated in step 3.

5. Replace the lost MBs with the candidate MBs by using the averaged calculated value.

Algorithm 5: Even-view B Frame Adaptive Time Domain EC (ATDEC) Algorithm

1. Find the locations of the lost MBs inside B frame.

2. Apply Scene Change Detection Algorithm [6], to decide "Is the received 3D MVC video fast or slow moving video?".

3. Select between the three ATDEC algorithms (FTRA or EBBMA or DMVEA) depending on step 2, as observed in Fig. 4 to find the matching pixels inside the previous and subsequent frames.

4. Apply step 3 of the Algorithm 1 for pixels inside the previous and subsequent frames.

Algorithm 6: Odd-view Inter-Frame Adaptive Time Inter-View Domain EC (ATIVDEC) Algorithm

1. Find the locations of the lost MBs inside B frame.

2. Check if Temporal information $>$ Spatial information or vice versa.

3. Apply steps 2 of Algorithm 4 and step 3 of Algorithms 5.

4. Search for the most matched candidates MVs and DVs to the lost MB within previous, subsequent, left and right frames.

5. Average DVs and MVs values of the candidates MBs.

6. Apply Scene Change Detection Algorithm [6], to check if Temporal information > Spatial information or vice versa.

7. Set appropriate coefficient values to averaged values of MVs and DVs ( avg (MVs) and avg (DVs), respectively) depending on Scene Change Detection Algorithm [6]:

If Temporal information < Spatial information

Else

- $\quad$ Candidate $\mathrm{MB}=1 / 3$ avg $(\mathrm{MVs})+2 / 3$ avg (DVs).

8. Replace the lost MBs with candidate MBs by using the weighted average of MVs and DVs calculated in last step.

\section{EXPERIMENTAL RESULTS}

To evaluate the performance of the proposed EC algorithms, we run some experiments on well-known video sequences [10]. For each sequence, the coded bit-streams are transmitted over a noisy channel with random Packet Loss Rates (PLRs) of 3\%, 5\%, 10\% or 20\%. The received bit-stream is then decoded by our proposed adaptive EC algorithms. It is assumed that the decoder can detect the locations of lost MBs.

Fig. 5. shows the subjective experimental results for the "Uli" sequence, which is a slow moving sequence. We select the $209^{\text {th }}$ intra-coded frame at channel PLR $=20 \%$. We recovered the $209^{\text {th }}$ intra-frame with the three proposed ASDEC, ATDEC and ASTDEC algorithms. The corresponding objective Peak Signal to Noise Ratio (PSNR) results for the same frame is shown in Fig. 6c at different channel PLRs. We observe that the hybrid ASTDEC has the best subjective and objective results compared to ATDEC and ASDEC algorithms, as the ASTDEC algorithm efficiently exploits the redundant information in both space and time domains. In Fig. 6a, we compare between the performance of deploying proposed ATDEC algorithm, (which adapts between FTRA, EBBMA, and DMVEA modes), and deploying either of FTRA or EBBMA or DMVEA algorithms only. Due to slow motion, FTRA algorithm gave better PSNR than DMVEA, which performed better than EBBMA algorithm, especially at low PLR. (The opposite was observed for the fast moving "Ballroom" video sequence). In Fig. 6b, we compare between the performance of deploying proposed ASDEC algorithm, which adaptively deploys either the WPAA or DIECA algorithm, and deploying either the WPAA or DIECA algorithm only. We 
observe that the DIECA algorithm gives higher objective results than WPAA algorithm. Fig. 6a and Fig. 6b show how our proposed adaptive EC algorithms correctly choose the component EC algorithm that best suits each error location, frame and video type.

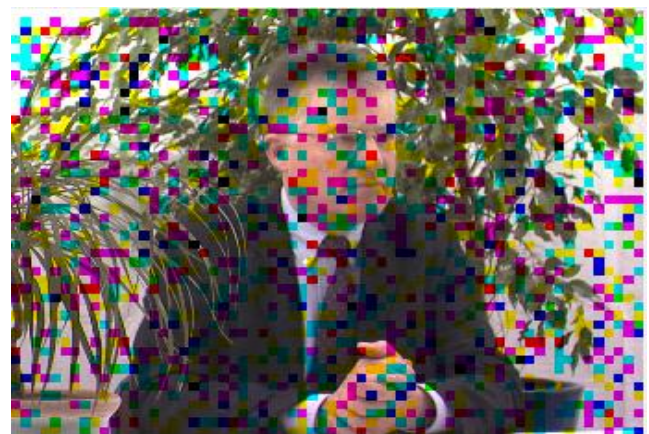

(a) $I_{209}$ intra-frame with PLR $=20 \%$.

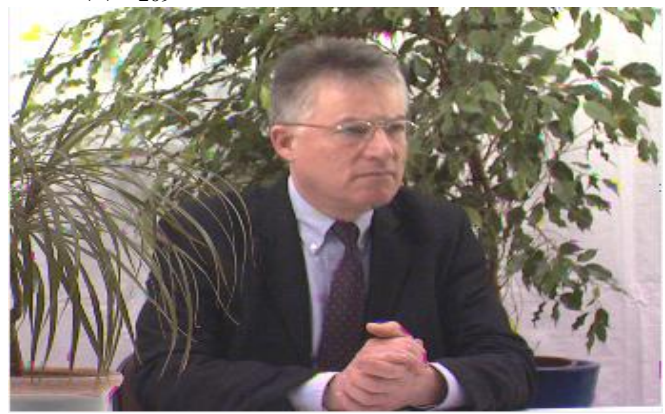

(c) Concealed $\mathrm{I}_{209}$ by ATDEC.

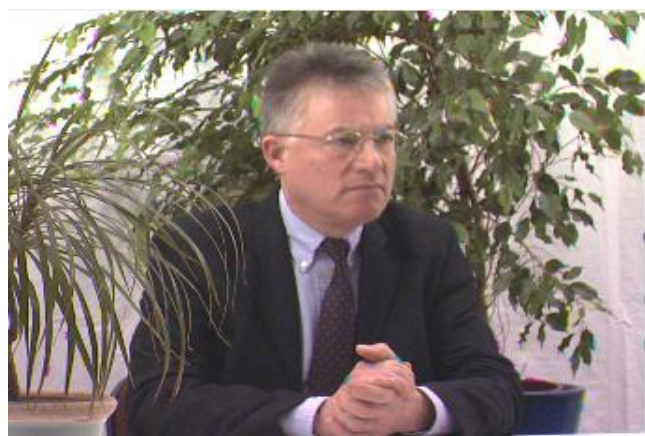

(b) Concealed $\mathrm{I}_{209}$ by ASDEC.

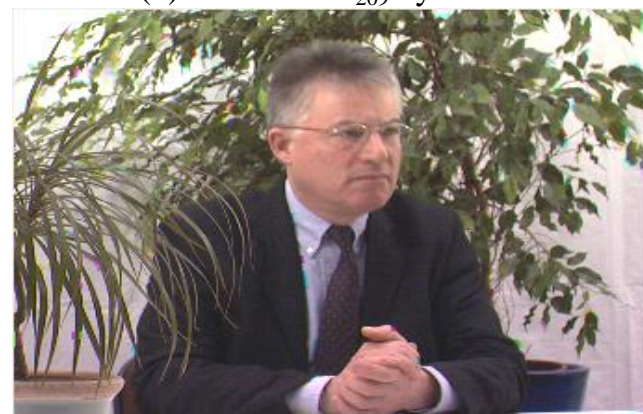

(d) Concealed $\mathrm{I}_{209}$ by ASTDEC.

Fig.5 Subjective simulation results for the selected $209^{\text {th }}$ intra-frame within the first view $\mathrm{S}_{\mathrm{o}}$ of the "Uli" sequence at channel PLR $20 \%$.

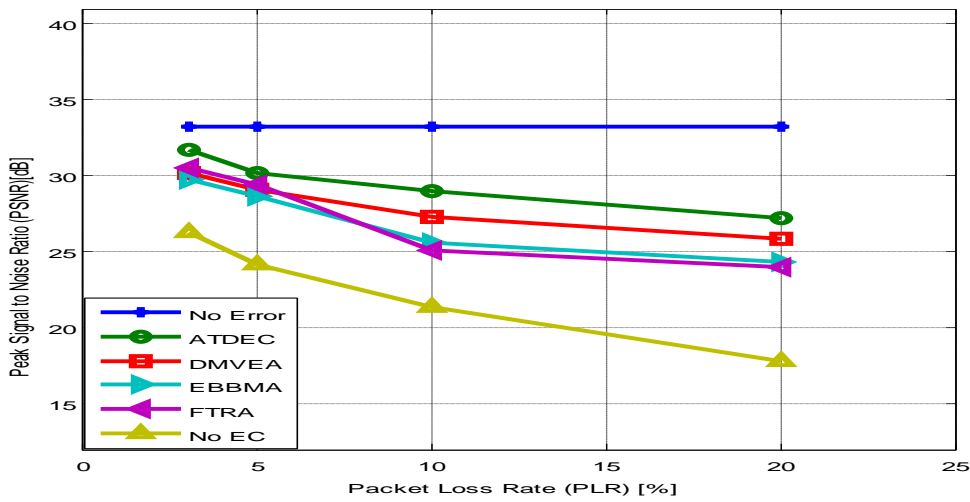

(a)

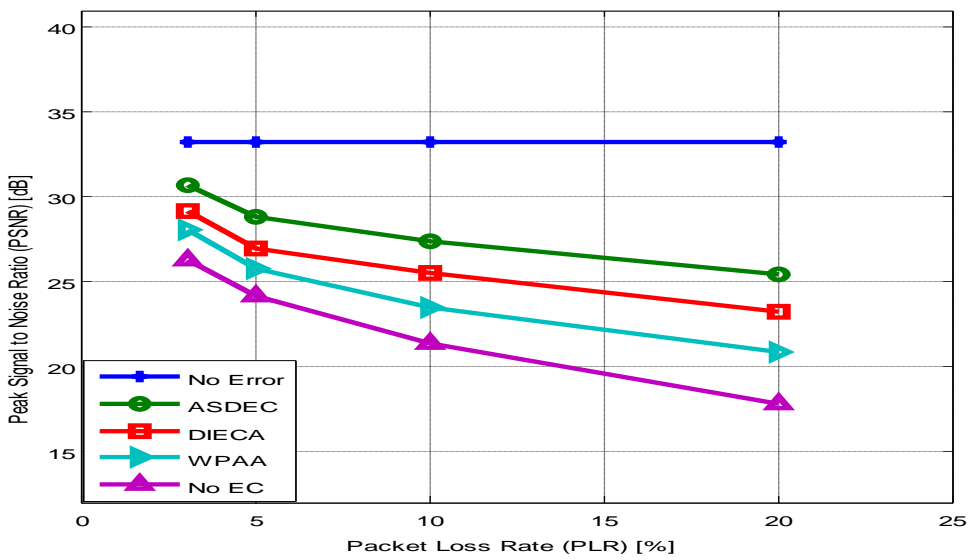

(b) 


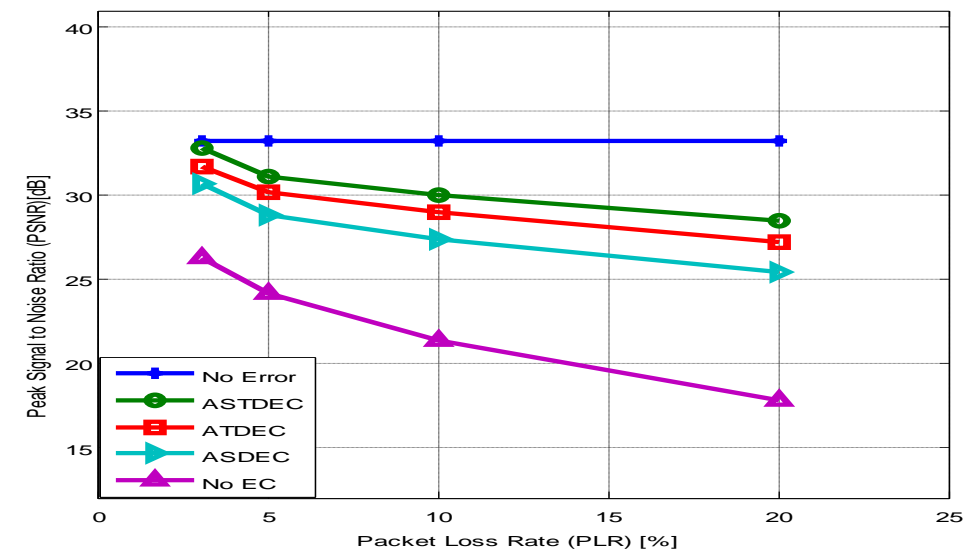

(c)

Fig.6 PSNR performance for "Uli" test sequence with different PLRs for the selected $209^{\text {th }}$ intra-frame, using intra-frames EC Algorithms 1, 2, and 3 and their proposed EC modes.

To demonstrate the effectiveness of the proposed adaptive $\mathrm{EC}$ algorithms for $\mathrm{P}$ and $\mathrm{B}$ inter-frames, the "Ballroom" sequence was tested with low complexity parameters [4] at the decoder which makes the EC process more practical. In the experimental tests, the $\mathrm{P}$ and $\mathrm{B}$ inter-view frames in views $\mathrm{S}_{1}$ and $\mathrm{S}_{2}$ are corrupted, with different random packet loss rates of 3\%,5\%,10\% and 20\%. The frames are recovered with the proposed adaptive EC Algorithms. Fig. 7, and Fig. 8 show the subjective simulation results of the odd and even views of the "Ballroom" sequence, respectively. It is noted that in Fig. $7 \mathrm{~b}$, the $209^{\text {th }}$ inter B frame in view $\mathrm{S}_{1}$ is concealed by using our proposed hybrid ATIVDEC algorithm without first error-concealing the intra-frame, while in Fig. $7 \mathrm{c}$ the intra-frame is also recovered using our EC algorithms. This demonstrates the importance of intra-frame EC. Fig. 8 shows the subjective results of the $209^{\text {th }}$ inter $\mathrm{P}$ frame in view $\mathrm{S}_{2}$ recovered using AIVDEC algorithm in Fig. 8b without deploying intra-frame EC and in Fig. 8c with using intra-frame EC.

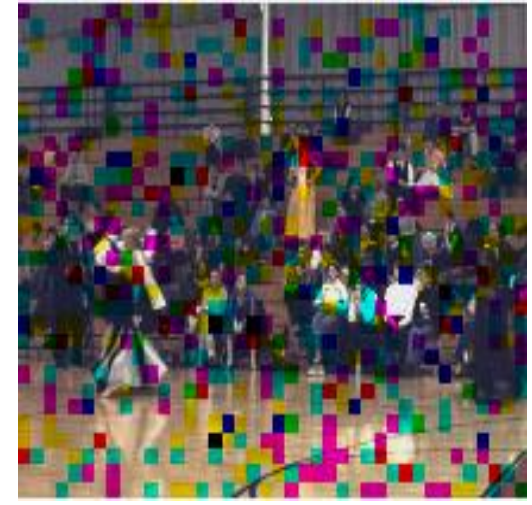

a) Inter $\mathrm{B}_{209}$ frame with PLR=20\%.

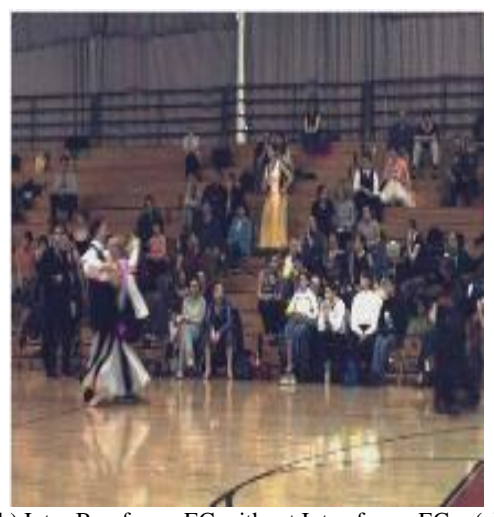

(b) Inter $\mathrm{B}_{209}$ frame $\mathrm{EC}$ without Intra-frame $\mathrm{EC}$. (c) Inter $\mathrm{B}_{209}$ frame $\mathrm{EC}$ with Intra-frame $\mathrm{EC}$.

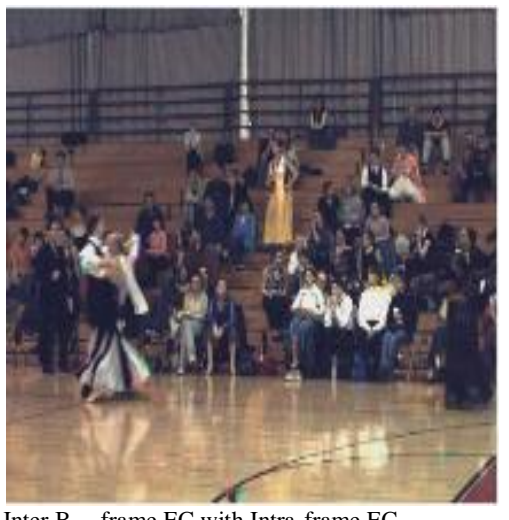

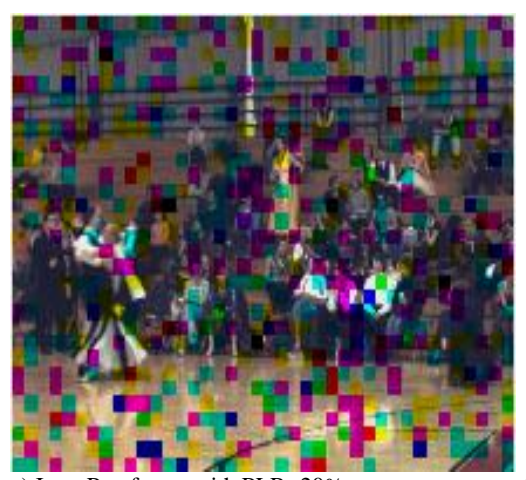

a) Inter $\mathrm{P}_{209}$ frame with PLR=20\%
$\mathrm{PLR}=20 \%$.

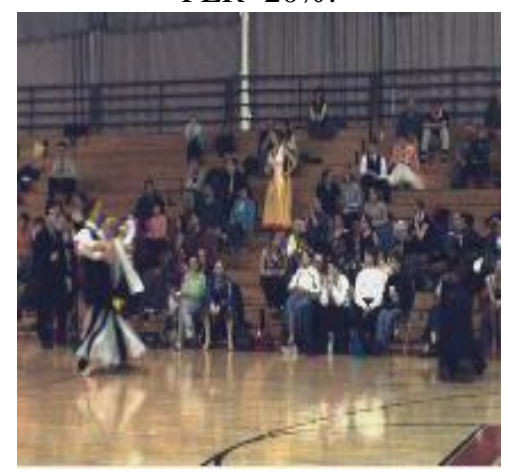

(b) Inter $\mathrm{P}_{209}$ frame $\mathrm{EC}$ without Intra-frame $\mathrm{EC}$

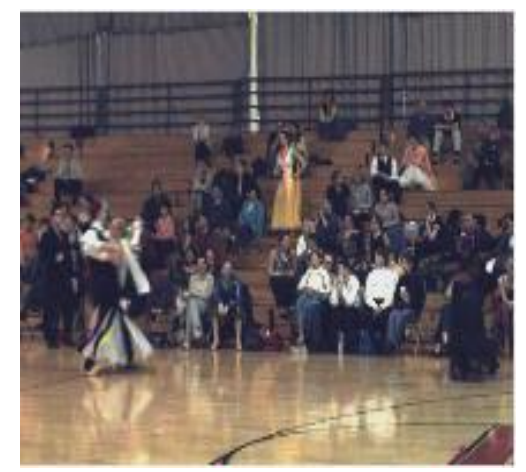

(c) Inter $\mathrm{P}_{209}$ frame $\mathrm{EC}$ with Intra-frame $\mathrm{EC}$. PLR $=20 \%$. 
Fig. 9. presents the objective simulation results of recovering odd views and even views of the 3D multi-view Ballroom video sequence. We again observe that intra-frame EC improves the performance of interframe EC. We also observe that the adaptive joint space-time-view EC algorithm proposed in this paper offers significant PSNR improvements over the non-adaptive joint algorithm previously proposed in [4].

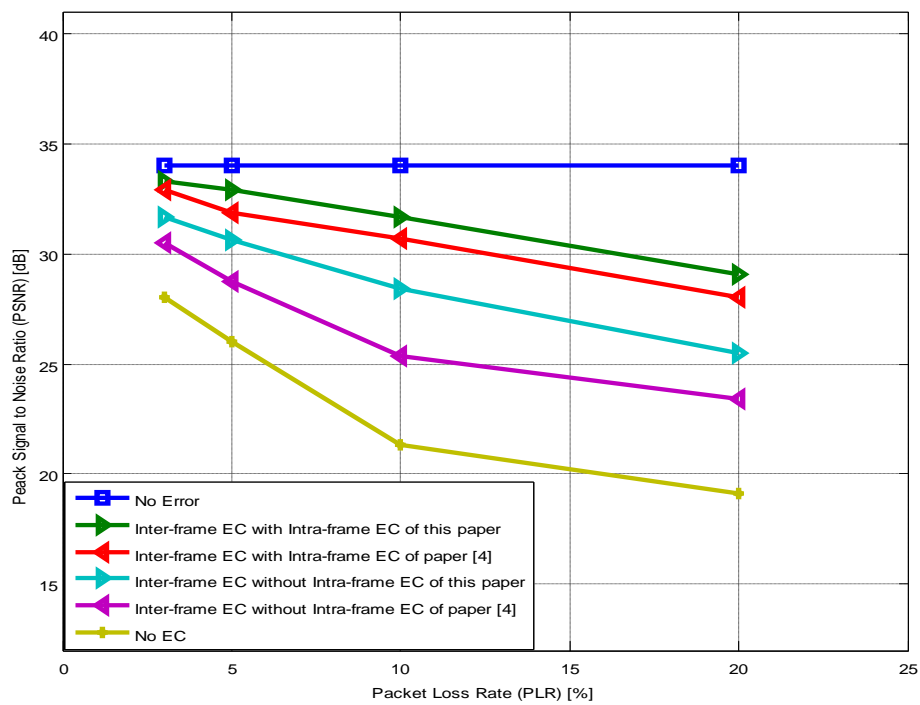

(a)

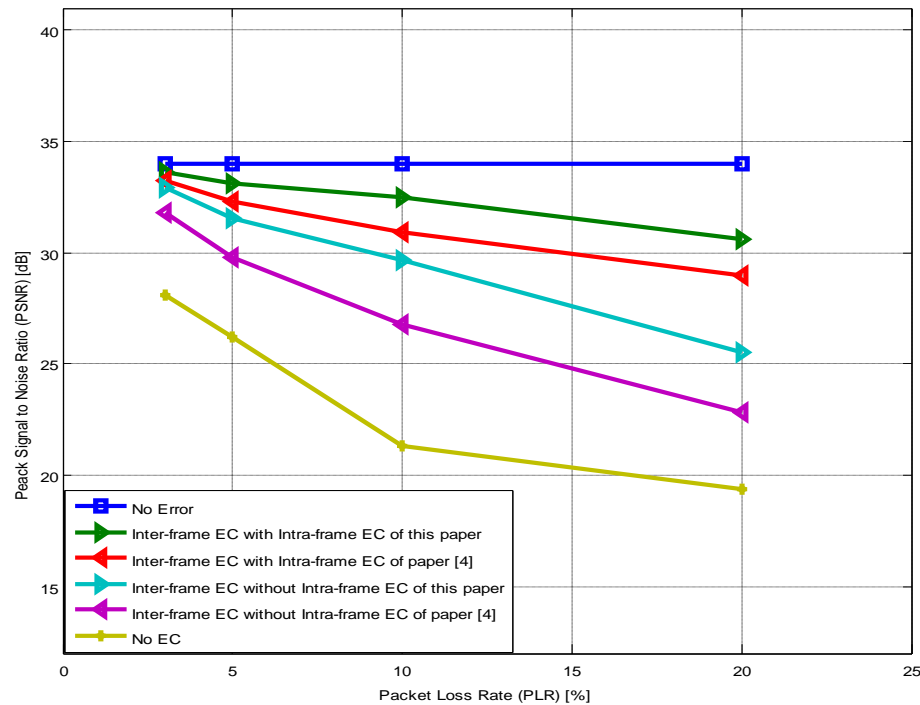

(b)

Fig. 9 PSNR performance for "Ballroom" test sequence with different PLR for inter-frames within (a) odd views and (b) even views.

\section{CONCLUSIONS}

In this paper, we have proposed low-complexity adaptive error concealment (EC) algorithms for 3D multi-view video coded (MVC) sequences corrupted by random channel errors. The main crux of our proposed algorithms is to adaptively and jointly utilize the spatial, temporal and inter-view correlations in MVC sequences for EC of both intra-frames and inter-frames. The proposed EC algorithm adapts to the video speed as well as to the error locations. Our experimental results show that our proposed adaptive hybrid time domain, space domain and inter-view domain EC algorithms are significantly superior to conventional EC algorithms that exploit correlation in only the space domain or only the time domain, e.g. [3, 6, 7, 8]. Our results demonstrate the importance of EC of intra-frames as it affects the quality of inter-frames error-concealment as well. We also show that the enhanced adaptive EC algorithm of this paper achieves better subjective video quality than the non-adaptive space-time-view algorithm previously proposed in [4], as well significant gain in objective PSNR. We conclude that the adaptive joint space-time-view EC algorithms proposed in this paper can conceal errors and lost MBs of MVC intra-frames and inter-frames efficiently, and delivering high quality 3D multi-view video. 


\section{REFERENCES}

[1] K. Song, T. Chung, Y. Oh, and C.-S. Kim, Error concealment of multi-view video sequences using inter-view and intra-view correlations, J. Vis. Comun. Image Represent., vol. 20, pp. 281-292, May 2009.

[2] P. Nasiopoulos, L. Coria-Mendozal, H. Mansour, and A. Golikeri, An improved error concealment algorithm for intra-frames in H. 264/AVC, IEEE International Symposium on Circuits and Systems, ISCAS. IEEE, 2005, pp. 320-323.

[3] T. Chung, K. Song, and C. Kim, Error concealment techniques for multi-view video sequences, Proceedings of the multimedia 8th Pacific Rim conference on Advances in multimedia information processing. Springer-Verlag, 2007, pp. 619-627.

[4] W. ElShafai, B. Hrusovsky, M. El-Khamy, and M. El-Sharkawy, Joint Space-Time-View error concealment algorithms for 3D MultiView video, 2011 18th IEEE International Conference on Image Processing (IEEE ICIP2011), Brussels, Belgium, Sep. 2011, pp. 2249-2252.

[5] B. Hrusovsky, J. Mochnac, and S. Marchevsky, Temporal-spatial error concealment algorithm for intra-frames in H. 264/AVC coded video, 2010 20th International Conference Radioelektronika. IEEE, 2010, pp. 1-4

[6] J. Seiler and A. Kaup, Adaptive joint spatio-temporal error concealment for video communication, 2008 IEEE 10th Workshop on Multimedia Signal Processing. IEEE, 2008, pp. 229-234.

[7] P. Merkle, A. Smolic, K. Muller, and T. Wiegand, Efficient prediction structures for multiview video coding, Circuits and Systems for Video Technology, IEEE Transactions on, vol. 17, no. 11, pp. 1461-1473, 2007.

[8] M. Hwang, J. Kim, D. Duong, and S. Ko, Hybrid temporal error concealment methods for block-based compressed video transmission, IEEE Transactions on Broadcasting, vol. 54, no. 2, pp. 198-207, 2008.

[9] Y. Chung, L. Chen, X. Chen, W. Yeh, and C. Bae, A novel intra-fame error concealment algorithm for H. 264 AVC, Third International Conference on Convergence and Hybrid Information Technology, 2008, ICCIT'08, vol. 1. IEEE, 2008, pp. 881-886.

[10] ftp://ftp.merl.com/pub/avetro/mvc-testseq. 